\title{
Breakup Density in Spectator Fragmentation
}

S. Fritz, ${ }^{(1)}$ C. Schwarz, ${ }^{(1)}$ R. Bassini, ${ }^{(2)}$ M. Begemann-Blaich, ${ }^{(1)}$ S.J. Gaff-Ejakov, ${ }^{(3)}$

D. Gourio, ${ }^{(1)}$ C. Groß, ${ }^{(1)}$ G. Immé, ${ }^{(4)}$ I. Iori, ${ }^{(2)}$ U. Kleinevoß, ${ }^{(1)}$ [a G.J. Kunde, ${ }^{(3)}$ [b

W.D. Kunze, ${ }^{(1)}$ U. Lynen, ${ }^{(1)}$ V. Maddalena, ${ }^{(4)}$ M. Mahi, ${ }^{(1)}$ T. Möhlenkamp, ${ }^{(5)}$

A. Moroni, ${ }^{(2)}$ W.F.J. Müller, ${ }^{(1)}$ C. Nociforo, ${ }^{(4)}$ B. Ocker, ${ }^{(6)}$ T. Odeh, ${ }^{(1)}$ 泡 F. Petruzzelli, ${ }^{(2)}$

J. Pochodzalla, ${ }^{(1)}$ [C] G. Raciti, ${ }^{(4)}$ G. Riccobene, ${ }^{(4)}$ F.P. Romano, ${ }^{(4)}$ A. Saija, ${ }^{(4)}$

M. Schnittker, ${ }^{(1)}$ A. Schüttauf, ${ }^{6}$ 《d] W. Seidel, ${ }^{(5)}$ V. Serfling, ${ }^{(1)}$ C. Sfienti, ${ }^{(4)}$

W. Trautmann, ${ }^{(1)}$ A. Trzcinski, ${ }^{(7)}$ G. Verde, ${ }^{(4)}$ A. Wörner, ${ }^{(1)}$ Hongfei Xi, ${ }^{(1)}$ and B. Zwieglinski ${ }^{(7)}$

(1) Gesellschaft für Schwerionenforschung, D-64291 Darmstadt, Germany

${ }^{(2)}$ Istituto di Scienze Fisiche, Università degli Studi di Milano and I.N.F.N., I-20133

Milano, Italy

(3) Department of Physics and Astronomy and National Superconducting Cyclotron

Laboratory, Michigan State University, East Lansing, MI 48824, USA

(4) Dipartimento di Fisica dell' Università and I.N.F.N., I-95129 Catania, Italy

${ }^{(5)}$ Forschungszentrum Rossendorf, D-01314 Dresden, Germany

(6) Institut für Kernphysik, Universität Frankfurt, D-60486 Frankfurt, Germany

(7) Soltan Institute for Nuclear Studies, 00-681 Warsaw, Hoza 69, Poland

\begin{abstract}
Proton-proton correlations and correlations of protons, deuterons and tritons with $\alpha$ particles from spectator decays following ${ }^{197} \mathrm{Au}+{ }^{197} \mathrm{Au}$ collisions at $1000 \mathrm{MeV}$ per nucleon have been measured with two highly efficient detector hodoscopes. The constructed correlation functions, interpreted within the approximation of a simultaneous volume decay, indicate a moderate expansion and low breakup densities, similar to assumptions made in statistical multifragmentation models.
\end{abstract}

PACS numbers: 25.70.Pq, 21.65.+f, 25.70.Mn, 25.75.Gz 
Expansion is a rather basic conceptual feature of the multifragmentation of heavy nuclei. A volume of about three to eight times that occupied at saturation density is assumed in the statistical models aiming at a phase space description of the multi-fragment breakups [1, 2]. Expansion also provides the link to the nuclear liquid-gas phase transition; only one third of the saturation value is expected for the critical density of nuclear matter [3]. The experimental confirmation of expansion to low breakup densities is therefore of the highest significance for the understanding and interpretation of the multifragmentation phenomenon [四].

In central collisions of heavy nuclei, rapid expansion is evident from the observation of radial collective flow [5]. For the fragment decay of spectators following collisions at relativistic energies, the case studied in this work, significant radial flow has not been observed [6]. Here evidence for expansion has been obtained, indirectly, from model comparisons. Models that assume sequential emission from nuclear systems at saturation density underpredict the fragment multiplicities while those assuming expanded breakup volumes yield satisfactory descriptions of the populated partition space [7, 8, 9, 10]. The disappearance of the Coulomb peaks in the kinetic-energy spectra of emitted light particles and fragments, associated with increasing fragment production, provides additional evidence consistent with volume emission from expanded systems [11, 12]. There are also other dynamical and statistical observables that have been interpreted as evidence for expansion in recent papers [13, 14, 15].

Interferometry-type methods permit experimental determinations of the breakup volume or, more precisely, of the space-time locations of the last collisions of the emitted products [16]. In the nuclear regime, correlation functions for light charged particles, predominantly proton-proton correlations, have been widely explored for that purpose [17, 18]. Depending on the assumed reaction scenario and energy regime, both time scales and breakup radii have been deduced. The time scales for the decay of highly excited spectator nuclei produced at relativistic bombarding energies should be rather short [19], and we may expect that the correlation functions are mainly sensitive to the spatial extension of the source. More importantly, if we assume a rapid volume breakup of the system, the quantity of interest will be the local density, i.e. the mutual proximity of the nascent fragments and light particles. These densities are obtained in the limit of assuming a zero-lifetime in the source analysis.

In this Letter, we present the results of correlation measurements for spectator decays following collisions of ${ }^{197} \mathrm{Au}+{ }^{197} \mathrm{Au}$ at a bombarding energy of $1000 \mathrm{MeV}$ per nucleon. Besides proton-proton coincidences, also coincidences of protons, deuterons, and tritons with $\alpha$ particles were measured and correlation functions were constructed. For their quantitative interpretation, it is assumed that they are dominated by the effect of finalstate interactions. The results are found to be consistent with low breakup densities with values close to those assumed in the statistical multifragmentation models.

Beams of ${ }^{197} \mathrm{Au}$ with incident energy $1000 \mathrm{MeV}$ per nucleon were provided by the heavy- 
ion synchrotron SIS and directed onto targets of $25-\mathrm{mg} / \mathrm{cm}^{2}$ areal thickness. Two multidetector hodoscopes, consisting of a total of $160 \mathrm{Si}-\mathrm{CsI}(\mathrm{Tl})$ telescopes in closely-packed geometry, were placed on opposite sides with respect to the beam axis. The angular range $\theta_{\text {lab }}$ from $122^{\circ}$ to $156^{\circ}$ was chosen with the aim of selectively detecting the products of the target-spectator decay. Each telescope consisted of a $300-\mu \mathrm{m}$ Si detector with 30 x $30 \mathrm{~mm}^{2}$ (96 detectors) or $25 \times 25 \mathrm{~mm}^{2}$ (64 detectors) active area, followed by a 6 -cm long $\mathrm{CsI}(\mathrm{Tl})$ scintillator with photodiode readout. The distance to the target was about $60 \mathrm{~cm}$.

The products of the projectile decay were measured with the time-of-flight wall of the ALADIN spectrometer [6] and the quantity $Z_{\text {bound }}$ was determined event-by-event. $Z_{\text {bound }}$ is defined as the sum of the atomic numbers $Z_{i}$ of all projectile fragments with $Z_{i} \geq 2$. It reflects the variation of the size of the primary spectator nuclei and is inversely correlated with its excitation energy. Because of the symmetry of the collision system, the mean values of $Z_{\text {bound }}$ for the target and the projectile spectators within the same event class have been assumed to be identical.

Examples of correlation functions constructed for the four types of coincidences p-p, p- ${ }^{4} \mathrm{He}$, d- ${ }^{4} \mathrm{He}$, and t- ${ }^{4} \mathrm{He}$ are shown in Fig. 1, sorted into four bins of $Z_{\text {bound }}$ as indicated. The uncorrelated yields were obtained with the technique of event-mixing and normalized in the range of large relative momenta $q \geq 70 \mathrm{MeV} / \mathrm{c}(\mathrm{p}-\mathrm{p})$ and $q \geq 150 \mathrm{MeV} / \mathrm{c}$ (p- $\alpha$, d- $\alpha$, and $\mathrm{t}-\alpha$ ). In the off-line analysis, thresholds were set at $E_{l a b} \geq 20 \mathrm{MeV}$ for all particles $\mathrm{p}, \mathrm{d}, \mathrm{t}$, and $\alpha$ and all hodoscope detectors. The shapes of the correlation functions are sensitive to the threshold. For p-p, e.g., the suppression at small $q$ tends to disappear if the threshold is set at $E_{l a b} \geq 10 \mathrm{MeV}$ or lower, presumably as a result of increasing contributions from evaporation and sequential decay. These long-lifetime components are suppressed if higher thresholds are chosen. The pairs of particles were also requested to be detected in the same hodoscope in order to avoid correlation effects that appear at large $q$ and are believed to be due to a collective (sidewards) motion of the proton-emitting source.

The p-p correlation functions are characterized by a depression at small relative momentum and by a weakly pronounced peak near relative momentum $q=20 \mathrm{MeV} / \mathrm{c}$, caused by the S-wave nuclear interaction and used for the quantitative interpretation. The three correlation functions of the hydrogen isotopes with $\alpha$ particles are dominated by the resonances corresponding to the ground state of ${ }^{5} \mathrm{Li}$ and by the $2.19-\mathrm{MeV}$ and $4.63-\mathrm{MeV}$ excited states of ${ }^{6} \mathrm{Li}$ and ${ }^{7} \mathrm{Li}$, respectively. The observed widths of the ${ }^{6} \mathrm{Li}$ and ${ }^{7} \mathrm{Li}$ peaks in the $\mathrm{d}-\alpha$ and $\mathrm{t}-\alpha$ correlation functions represent the experimental resolution which is mainly determined by the angular resolution following from the geometry of the detector hodoscopes. A striking feature of the data is the overall stability of the peak heights as a function of $Z_{\text {bound }}$ which indicates source extensions that do not change dramatically with impact parameter. The largest deviations of the peak height and of the overall shape of the correlation functions appear in the bin $60 \leq Z_{\text {bound }} \leq 79$, corresponding to the largest impact parameters.

The analysis of the p-p correlation functions was performed with the Koonin-Pratt 


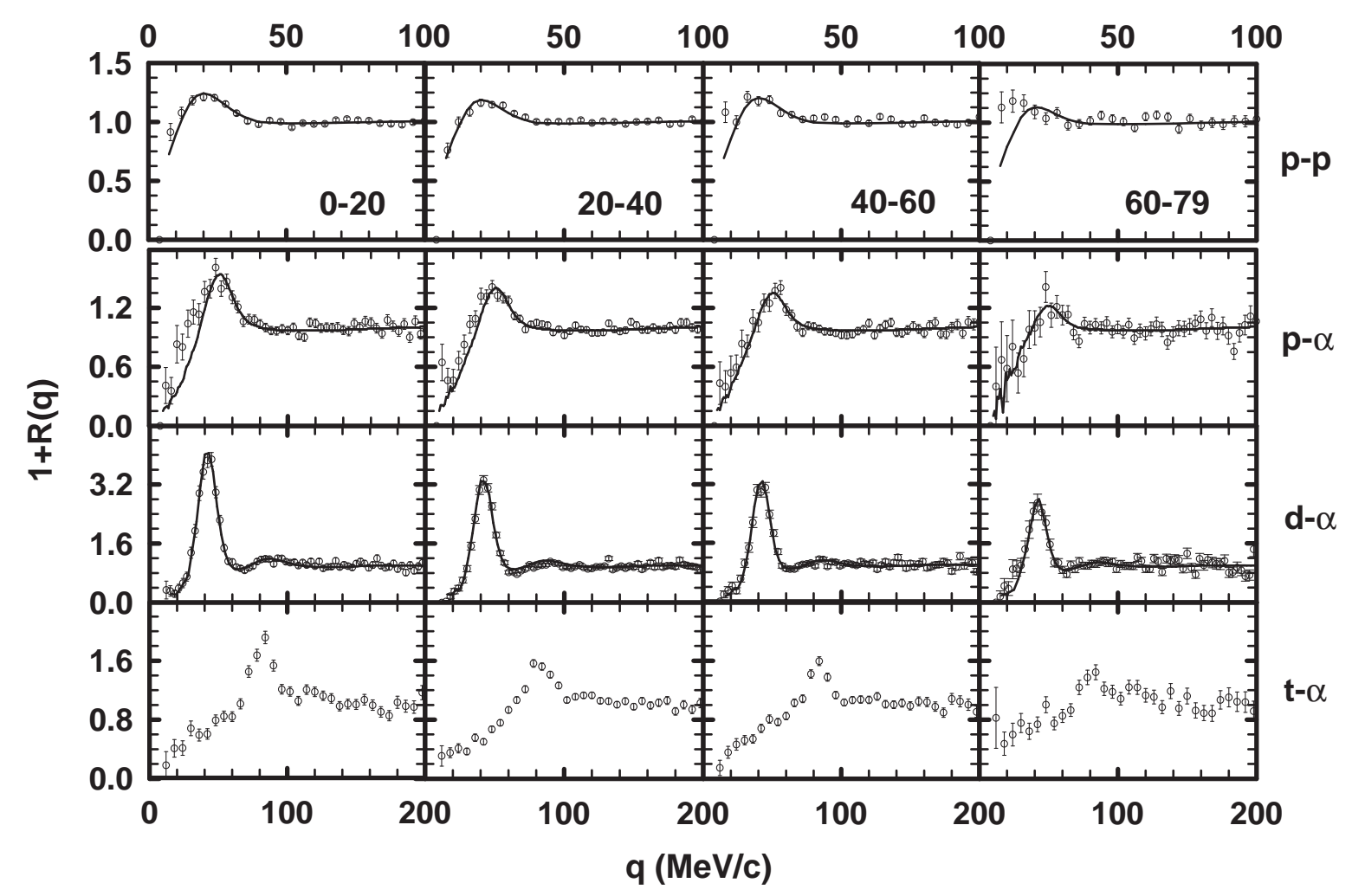

Figure 1: Correlation functions constructed for p-p, $\mathrm{p}-{ }^{4} \mathrm{He}, \mathrm{d}-{ }^{4} \mathrm{He}$, and t- ${ }^{4} \mathrm{He}$ coincidences (from top to bottom) for spectator decays following ${ }^{197} \mathrm{Au}+{ }^{197} \mathrm{Au}$ collisions at $1000 \mathrm{MeV}$ per nucleon. The data are sorted according to $Z_{\text {bound }}$ into four bins with limits given in the top panel of each column. The lines represent the results of the calculations used to extract source radii (see text). Note that the scales of the abscissa are different for the top row (p-p correlations) and for the remaining three rows of panels.

formalism [20] in the zero-lifetime limit. In this form, the analysis includes the effects of quantum statistics and of the mutual nuclear and Coulomb final-state interactions but it ignores the long-range Coulomb repulsion of the two protons from the emitting source. In order to assess the magnitude of the latter effect, classical Coulomb trajectory calculations were performed. In addition, calculations with the three-body quantum model of Lednicky et al. 21] were used to identify possible systematic uncertainties. A uniform sphere with radius $R$ and statistical momentum distributions corresponding to the measured kineticenergy spectra were assumed for the proton source; the model results are slightly dependent on the particle momenta through the applied experimental filter.

The quality of the obtained results and the sensitivity to the radius parameter $R$ is illustrated in Fig. 2 for the case $20 \leq Z_{\text {bound }} \leq 40$. With the filtered Koonin-Pratt calculations, the most satisfactory description of the data is obtained with $R=8.2 \mathrm{fm}$ (full line). The 


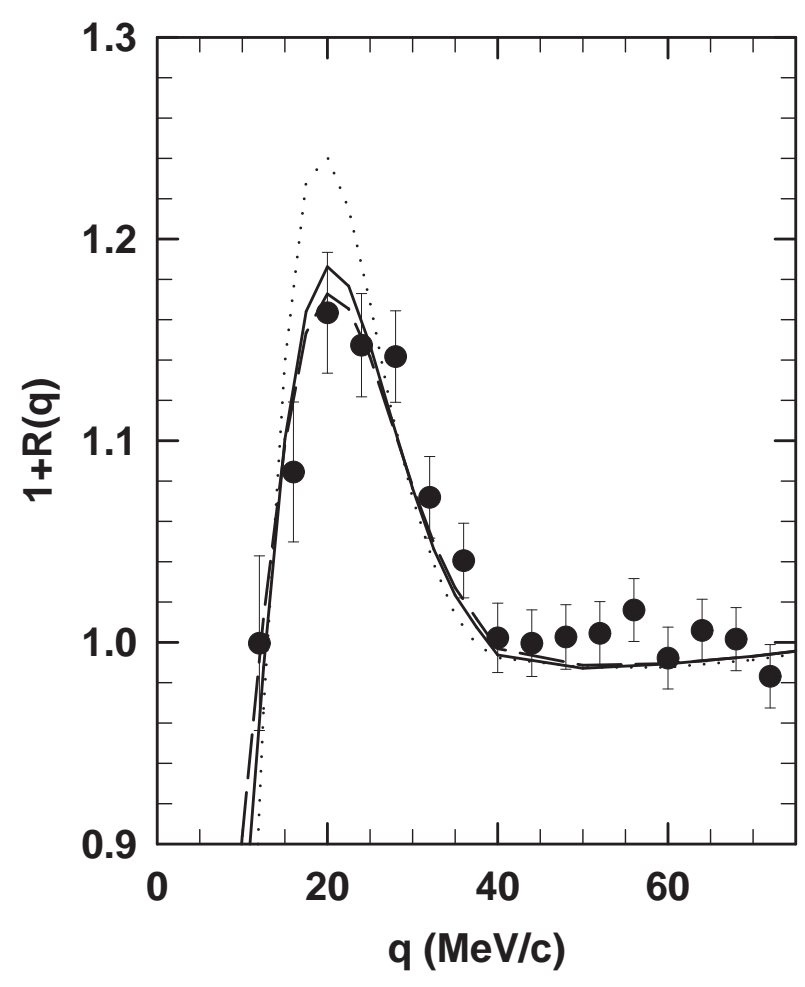

Figure 2: Proton-proton correlation function for the interval $20 \leq Z_{\text {bound }} \leq 40$ in comparison with the predictions of the Koonin-Pratt formalism before (dotted) and after applying the experimental filter (full line), and after correction for the Coulomb repulsion from the emitting source (dashed line, closely following the full line). The calculations were performed for a homogeneous spherical source with radius $R=8.2 \mathrm{fm}$.

height and the width of the resonance peak are rather well reproduced. For comparison, the results of the same calculation before filtering (dotted line) and after correction for the Coulomb repulsion from the source (dashed line) are also shown. The filtering effect is equivalent to a change of the radius by $\Delta R \approx 0.3 \mathrm{fm}$ whereas the modification caused by the charge of the emitting source is almost negligible. The latter is not unexpected in the present case, especially for the more central collisions which produce spectators of very moderate total charge (e.g. $\approx 45$ for $20 \leq Z_{\text {bound }} \leq 40$ ). In addition, with the assumption of a simultaneous volume break-up, only the charge inside the volume corresponding to the radial position of the particle was assumed to contribute to its acceleration. Correlation peaks of nearly the same height but with slightly smaller width were obtained with the three-body quantum model [21]. In the two-body approximation, the difference between the results obtained with the Koonin-Pratt and the Lednicky et al. formalisms is practically negligible. Therefore, and because of the reduced importance of the three-body Coulomb effect, the quantitative analysis of the measured p-p correlation functions was performed with the filtered Koonin-Pratt simulations.

The best fits, generated by minimizing $\chi^{2}$ within the range $10 \leq q \leq 35 \mathrm{MeV} / \mathrm{c}$ are shown in Fig. 1. The corresponding radii, listed in Table 1, are close to about $8 \mathrm{fm}$, up to nearly $9 \mathrm{fm}$ for the bin of largest $Z_{\text {bound }}$ which, however, seems to be afflicted with the largest uncertainties. These values are distinctly but not excessively larger than the radius $R=6.7 \mathrm{fm}$ of a gold nucleus at normal density $0.16 \mathrm{fm}^{-3}$. The quoted errors are purely 


\begin{tabular}{|l|c|c|c|c|}
\hline$Z_{\text {bound }}$ & $0-20$ & $20-40$ & $40-60$ & $60-79$ \\
\hline$<A>$ & 50 & 110 & 150 & 184 \\
\hline $\mathrm{p}-\mathrm{p}$ & $7.8 \pm 0.1$ & $8.2 \pm 0.2$ & $8.1 \pm 0.2$ & $8.8 \pm 0.6$ \\
& $(0.7)$ & $(1.4)$ & $(1.0)$ & $(3.2)$ \\
\hline $\mathrm{p}-\alpha$ & $9.5 \pm 0.4$ & $10.5 \pm 0.4$ & $10.9 \pm 0.3$ & $12.7 \pm 0.8$ \\
& $(3.3)$ & $(2.6)$ & $(0.8)$ & $(0.8)$ \\
\hline $\mathrm{d}-\alpha$ & $8.4 \pm 0.2$ & $9.3 \pm 0.1$ & $9.5 \pm 0.2$ & $10.5 \pm 0.2$ \\
& $(0.7)$ & $(1.2)$ & $(1.6)$ & $(0.3)$ \\
\hline
\end{tabular}

Table 1: Mean mass numbers $<A>$ and extracted source radii in units of $\mathrm{fm}$ for the indicated bins of $Z_{\text {bound }}$. The regions of relative momentum $q$ selected for the $\chi^{2}$ test are $10-35,30-70$, and $30-54 \mathrm{MeV} / \mathrm{c}$ for the $\mathrm{p}-\mathrm{p}, \mathrm{p}-\alpha$, and $\mathrm{d}-\alpha$ correlation functions, respectively. The numbers in brackets denote the minimum $\chi^{2}$ per degree of freedom.

statistical and were determined from the radii for which $\chi^{2}$ exceeds its minimum by an amount equal to the minimum value of $\chi^{2}$ per degree of freedom (i.e. for $\chi^{2}=\chi_{\min }^{2} \cdot n /(n-1)$ where $n$ is the number of data points included). The additional systematic uncertainty, mainly resulting from the arbitrariness of choosing the normalization interval and estimated to be about $0.5 \mathrm{fm}$, is much larger.

Correlation functions for proton pairs belonging to four intervals of the laboratory pair momentum $P_{\text {sum }}=\left|\vec{p}_{1}+\vec{p}_{2}\right|$, integrated over $Z_{\text {bound }}$, are shown in Fig. 3 . Only the data from the 96-element hodoscope were used. The combined effects of the energy threshold $E_{l a b} \geq 20 \mathrm{MeV}$, of the binning in pair momentum, and of the finite solid-angle acceptance of the detector hodoscope cause the limitation of the populated $q$ range for the bins of smaller $P_{\text {sum. }}$. A normalization interval $70 \leq q \leq 90 \mathrm{MeV} / \mathrm{c}$ was therefore chosen for these correlation functions. The same effects, perhaps including some collectivity due to a small but finite source motion, may cause the slight decrease towards larger $q$ that is not observed in the momentum-integrated correlation functions (cf. Fig. 1). We observe, however, that the peak height at $q \approx 20 \mathrm{MeV} / \mathrm{c}$ relative to the uncorrelated background at $q>40 \mathrm{MeV} / \mathrm{c}$ is virtually identical in all four cases. This is consistent with what is expected for the ideal situation of a purely statistical source, confirming that effects caused by collective radial motion or emission times related to the temporal evolution of an expanding source should be small for spectator decays.

The choice of a threshold of $E_{l a b} \geq 20 \mathrm{MeV}$, on the other hand, raises the question of what reaction stage is mainly represented by the obtained correlation data. Light-particle spectra from the same experiment, measured with high-resolution telescopes at backward angles, give evidence for emission prior to the final breakup stage [22]. This was concluded from the comparison of the slope temperatures and multiplicities to the predictions of the 


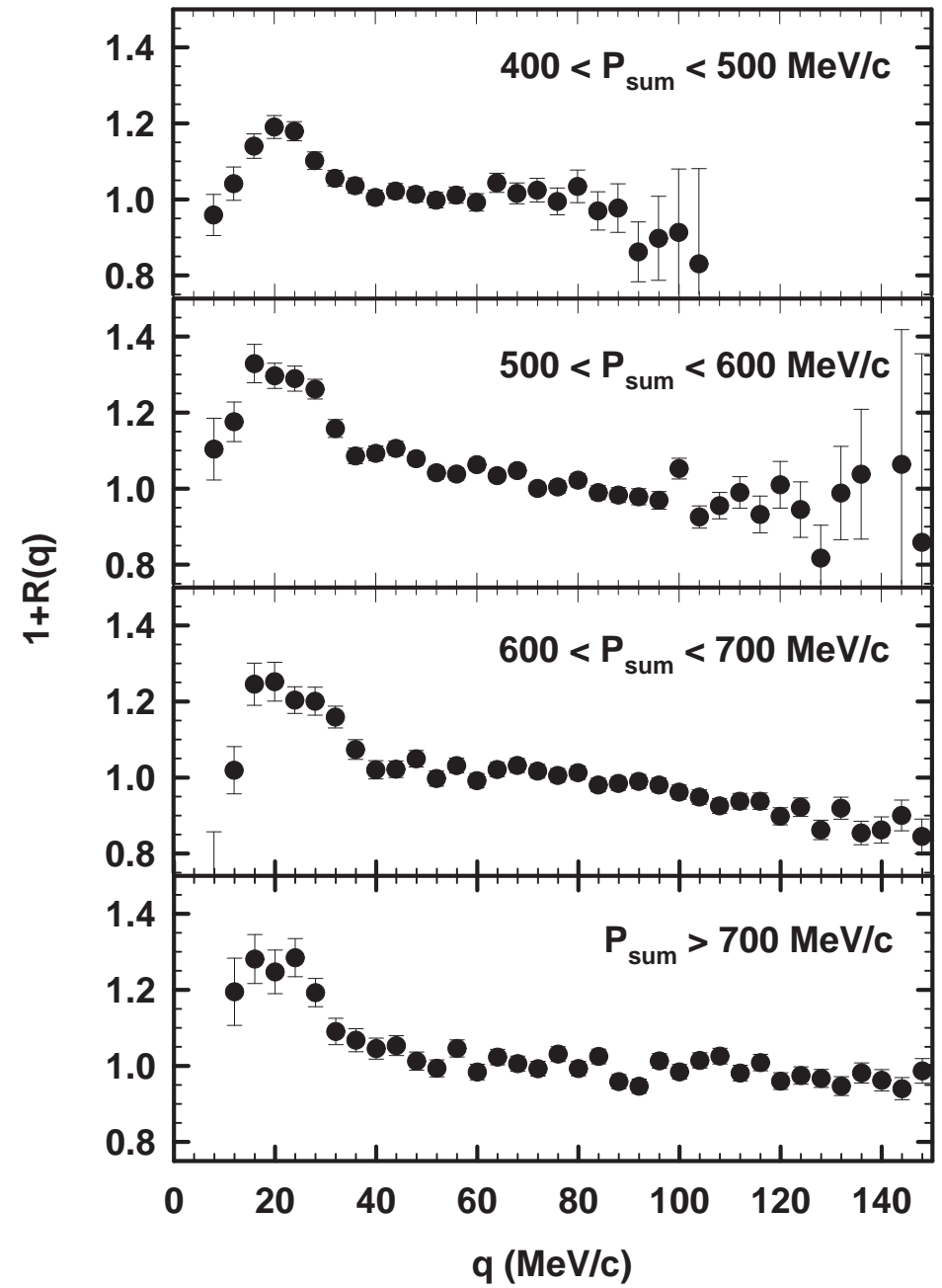

Figure 3: Proton-proton correlation functions, integrated over $Z_{\text {bound, for four intervals of the }}$ absolute value of the proton pair momentum. The interval $70 \leq$ $q \leq 90 \mathrm{MeV} / \mathrm{c}$ was used for normalization.

statistical multifragmentation model. Distinct components of equilibrium yields and of faster particles, termed pre-equilibrium or first stage, have also been identified by other groups in data for comparable reactions [13, 23]. In all these cases the main parts of the low-energy equilibrium components are below the present threshold. Long-lifetime components are thus excluded from the analysis so as to preserve the sensitivity of the correlation function to the initial spatial dimensions. On the other hand, pre-equilibrium or pre-breakup particles as they are scattered from the forming spectator matter may also contribute to an interferometric picture that reflects the extension of the latter.

Besides the p-p correlations also the $\mathrm{p}-\alpha$ and $\mathrm{d}-\alpha$ correlations were used to determine breakup radii by comparing them to the numerical results of Boal and Shillcock [24]. The calculated correlation functions which in their work are given for a discrete set of source radii were interpolated, and a Monte Carlo procedure was used for an event-by-event simulation of the effects of multiple scattering in the target and of the spatial and energy 


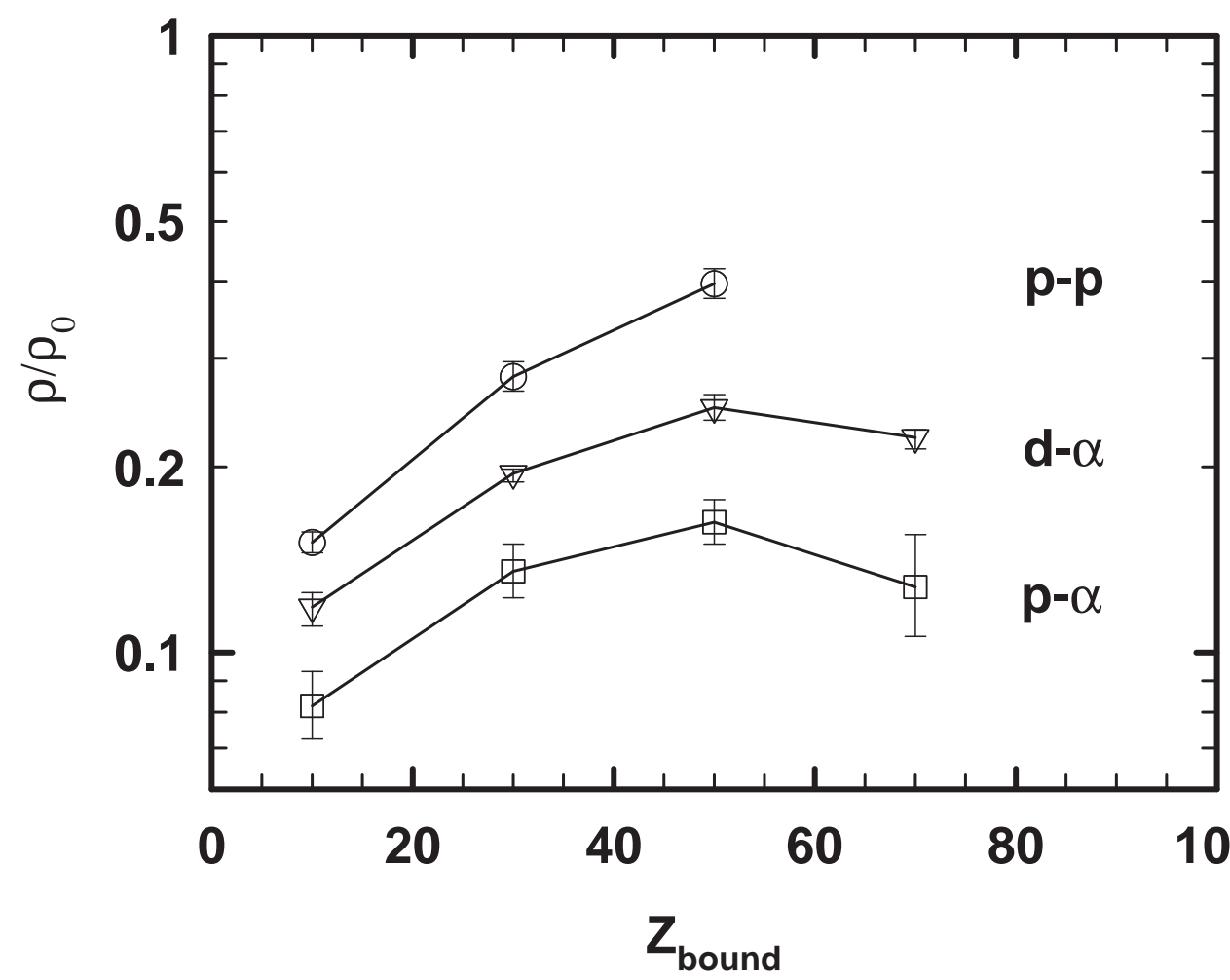

Figure 4: Breakup density $\rho / \rho_{0}$ as deduced from the p-p, $\mathrm{p}-{ }^{4} \mathrm{He}$, and $\mathrm{d}-{ }^{4} \mathrm{He}$ correlation functions. The rapid decrease of the density with decreasing $Z_{\text {bound }}$ reflects the changing mass of the spectator source. The error bars represent the statistical uncertainty of the radii as given in the table (see text). Note the logarithmic ordinate scale.

resolution of the detection system [25]. The Coulomb acceleration by the emitting source was also here neglected. This seems justified for $\mathrm{d}-\alpha$ because the effects should not be much larger than in the p-p case. For p- $\alpha$, the different charge-to-mass ratios of the two particles may be expected to cause larger distortions but, at the same time, the finite lifetimes of these resonances may reduce them considerably. The resulting fits to the data are rather satisfactory (Fig. 1). The corresponding radii are about 1 to $2 \mathrm{fm}$ larger than those deduced from the p-p correlations (Table 1). We also observe a slightly stronger variation with the pair momentum than in the p-p case. For p- $\alpha$, e.g., the peak of the resonance grows from about 1.3 to 1.6 as the pair momentum is varied from $P_{\text {sum }} \leq 800$ $\mathrm{MeV} / \mathrm{c}$ to $P_{\text {sum }}>1000 \mathrm{MeV} / \mathrm{c}$ (result after integration over $Z_{\text {bound }}$ ).

Densities were calculated by dividing the number of spectator constituents, taken from the calorimetric analyses of Refs. [26, 27] and listed in Table 1, by the source volume. The densities vary considerably with centrality (Fig. 4) even though the radii are approximately constant. This is caused by the varying spectator masses which, in excellent agreement with the prediction of the geometric participant-spectator model [28], decrease with increasing 
centrality almost in proportion to $Z_{\text {bound }}$.

In the $\mathrm{p}$-p case, the mean relative densities decrease from $\rho / \rho_{0}=0.4$ for the nearperipheral to below $\rho / \rho_{0}=0.2$ for the most central collisions. These values compare well with the densities assumed in the statistical multifragmentation model, including their variation with centrality. In the model, the mean density changes as a function of the multiplicity if a fixed socalled crack width is used as criterion for the placement of fragments inside the breakup volume [2]. For the most peripheral bin, the interpretation of the shape of the measured p-p correlations is somewhat uncertain (cf. Fig. 1), and the corresponding density has not been plotted. For $\mathrm{p}-\alpha$ and $\mathrm{d}-\alpha$, the fits are satisfactory for the largest $Z_{\text {bound }}$ but correspond to rather large radii (Table 1). The densities are therefore low which is somewhat unexpected as here the production of highly excited heavy residues is the dominant reaction channel [6]. Obviously, the assumptions of a homogeneous spherical source and of a rapid volume breakup are less well justified in this case which causes difficulties for the interpretation of the obtained density values.

The differences between the results for $\mathrm{p}-\mathrm{p}$ and for the resonances involving $\alpha$ particles are statistically significant but their origin is not clear at present. Besides the systematic uncertainties of the employed formalisms, there is also the possibility of differences in the scattering cross sections; larger cross sections will cause larger apparent source sizes. An interesting connection exists between the large radii derived for the lithium states and the limitation of the temperatures deduced from their relative populations [29]. A late emission of these resonances could explain both observations.

In summary, correlation functions constructed from proton-proton coincidences and from coincidences between protons, deuterons, or tritons with $\alpha$ particles consistently show that the breakup volume does not appreciably change with impact parameter even though the spectator mass varies considerably. A quantitative analysis, in the limit of zero lifetime of the source, yields results that are consistent with a very moderate radial expansion. The deduced breakup densities are rather low as assumed in the statistical model scenarios. The variation of the density with impact parameter is caused by the changing spectator mass. With regard to the method, it has become clear that the systematic uncertainties of deriving densities from correlation functions may be large, in particular for the resonances involving $\alpha$ particles for which the formalism is not yet as much advanced. It seems, however, that the spectator decay at relativistic energies may represent a particularly favorable case because the source charge is moderate, collective motion is nearly nonexistent, and with the choice of high energy thresholds long-lifetime components may have been efficiently excluded from the analysis.

The authors would like to thank R. Lednicky for making his code available to them and W.A. Friedman for fruitful discussions. M.B., J.P., and C.S. acknowledge the financial support of the Deutsche Forschungsgemeinschaft under the Contract No. Be1634/1-1, Po256/2-1, and Schw510/2-1, respectively. This work was supported by the European Community under contract ERBFMGECT950083. 


\section{References}

[a] Present address: Fachbereich Physik, Bergische Universität, D-42119 Wuppertal, Germany

[b] Present address: Dept. of Physics, Yale University, New Haven CT 06512, USA

[c] Present address: Max-Planck-Institut für Kernphysik, D-69117 Heidelberg, Germany

[d] Present address: Max-Planck-Institut für Physik, D-80805 München, Germany

[1] D.H.E. Gross, Rep. Prog. Phys. 53, 605 (1990)

[2] J.P. Bondorf et al., Phys Rep. 257, 133 (1995)

[3] See, e.g., H. Jaqaman et al., Phys. Rev. C 27, 2782 (1983)

[4] For a recent review see L.G. Moretto and G.J. Wozniak, Ann. Rev. Nucl. Part. Science 43, 379 (1993)

[5] W. Reisdorf and H.G. Ritter, Ann. Rev. Nucl. Part. Science 47, 663 (1997)

[6] A. Schüttauf et al., Nucl. Phys. A 607, 457 (1996)

[7] D.R. Bowman et al., Phys. Rev. Lett. 67, 1527 (1991)

[8] J. Hubele et al., Phys. Rev. C 46, R1577 (1992)

[9] K. Hagel et al., Phys. Rev. C 50, 2017 (1994)

[10] A.S. Botvina et al., Nucl. Phys. A 584, 737 (1995)

[11] U. Milkau et al., Phys. Rev. C 44, R1242 (1991)

[12] K. Kwiatkowski et al., Phys. Rev. Lett. 74, 3756 (1995)

[13] J.A. Hauger et al., Phys. Rev. C 57, 764 (1998)

[14] M. Begemann-Blaich et al., Phys. Rev. C 58, 1639 (1998)

[15] S.P. Avdeyev et al., Eur. Phys. J. A 3, 75 (1998)

[16] For a recent review and for references to earlier reviews, see D. Ardouin, Int. J. Mod. Phys. E 6, 391 (1997)

[17] J. Pochodzalla et al., Phys. Rev. C 35, 1695 (1987) 
[18] W.G. Gong et al., Phys. Rev. C 43, 781 (1991)

[19] G. Wang et al., Phys. Rev. C 57, R2786 (1998)

[20] S. Pratt and M.B. Tsang, Phys. Rev. C 36, 2390 (1987)

[21] R. Lednicky et al., preprint SUBATECH-94-19, Nantes 1994; L. Martin et al., Nucl. Phys. A 604, 69 (1996)

[22] Hongfei Xi et al., Z. Phys. A 359, 397 (1997)

[23] K. Kwiatkowski et al., Phys. Lett. B 423, 21 (1998)

[24] D.H. Boal and J.C. Shillcock, Phys. Rev. C 33, 549 (1986)

[25] C. Schwarz et al., Proceedings of the International Workshop XXVII on Gross Properties of Nuclei and Nuclear Excitations, Hirschegg, Austria, edited by H. Feldmeier et al. (GSI, Darmstadt, 1999) p. 168

[26] J. Pochodzalla et al., Phys. Rev. Lett. 75, 1040 (1995)

[27] C. Groß, PhD thesis, Universität Frankfurt, 1998

[28] J. Gosset et al., Phys. Rev. C 16, 629 (1977)

[29] V. Serfling et al., Phys. Rev. Lett. 80, 3928 (1998) 